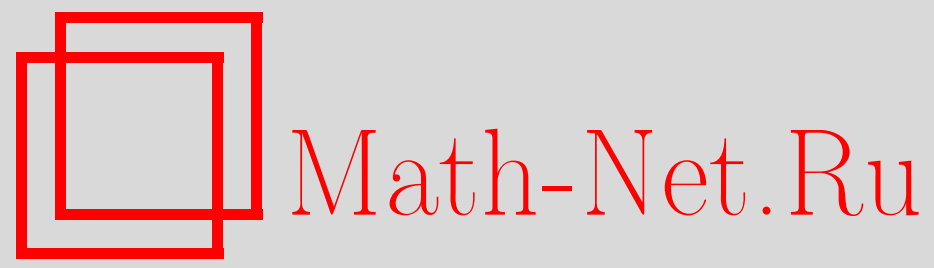

О. В. Камловский, Метод тригонометрических сумм для исследования частот $r$ грамм в старших координатных последовательностях линейных рекуррент над кольцом $\mathbb{Z}_{2^{n}}$, Матем. вопр. криптогр., 2010, том 1, выпуск 4, 33-62

DOI: https://doi.org/10.4213/mvk20

Использование Общероссийского математического портала Math-Net.Ru подразумевает, что вы прочитали и согласны с пользовательским соглашением

http://www.mathnet.ru/rus/agreement

Параметры загрузки:

IP : 54.147 .182 .235

26 апреля 2023 г., 15:35:23 


\title{
Метод тригонометрических сумм для исследования частот $r$-грамм в старших координатных последовательностях линейных рекуррент над кольцом $\mathbb{Z}_{2^{n}}$
}

\author{
О. В. Камловский \\ ООО «Центр сертификационных исследований», г. Москва
}

Получено 22.IV.2010

В работе методом тригонометрических сумм исследуются частотные характеристики старших координатных последовательностей (в произвольном координатном множестве) линейных рекуррент над кольцом $\mathbb{Z}_{2^{n}}$, характеристические многочлены которых, будучи приведенными по модулю два, неприводимы над полем $G F(2)$.

Ключевые слова: тригонометрические суммы, оценки сумм характеров, линейные рекуррентные последовательности, старшие координатные последовательности, частотные характеристики последовательностей

Exponential sums method for frequencies of most significant bit $r$-patterns in linear recurrent sequences over $\mathbb{Z}_{2^{n}}$

O. V. Kamlovskii

LLC "Certification Research Center", Moscow

Abstract. By means of exponential sums method we investigate distributions of $r$-patterns in the most significant bit of linear recurrent sequences over $\mathbb{Z}_{2^{n}}$ such that their characteristic polynomials reduced to mod 2 are irreducible over $G F(2)$.

Key words: exponential sums, character sums estimates, linear recurrent sequences, most significant bit sequences, $r$-patterns distributions

Citation: Mathematical Aspects of Cryptography, 2010, vol. 1, no. 4, pp. 33-62 (Russian). 


\section{1. Введение}

Подмножество $K=\left\{k_{0}, k_{1}\right\}$ множества $\mathbb{Z}_{2^{n}}$ назовем координатным множеством кольца $\mathbb{Z}_{2^{n}}$ (см., например, [1]), если элементы $k_{0}$ и $k_{1}$, рассматриваемые как целые числа, имеют различную четность. Примером координатного множества кольца $\mathbb{Z}_{2^{n}}$ является двоичное координатное множество $K=\{0,1\}$. Число всех координатных множеств кольца $\mathbb{Z}_{2^{n}}$ равно $2^{2(n-1)}$. Если $K-$ координатное множество кольца $\mathbb{Z}_{2^{n}}$, то каждый элемент $a$ этого кольца однозначно представим в виде

$$
a=a_{0}+2 a_{1}+2^{2} a_{2}+2^{3} a_{3}+\ldots+2^{n-1} a_{n-1},
$$

где $a_{i}$ - элемент из множества $K$ для всех $i=0,1, \ldots, n-1$. Отметим, что в равенстве (1) операции сложения и умножения рассматриваются как операции в кольце $\mathbb{Z}_{2^{n}}$. Равенство (1) будем называть координатным представлением элемента $a$ в координатном множестве $K$, а элемент $a_{n-1}$ будем называть старшей координатой элемента $a$ в координатном множестве $K$.

Всюду в дальнейшем будем считать, что координатное множество $K$ кольца $\mathbb{Z}_{2^{n}}$ имеет вид $K=\left\{k_{0}, k_{1}\right\}$, где $k_{0}-$ четное число, а $k_{1}-$ нечетное число. Рассмотрим отображение $\varkappa_{n-1}^{K}$ из множества $\mathbb{Z}_{2^{n}}$ в множество $K$, задаваемое для всех $a \in \mathbb{Z}_{2^{n}}$ по правилу

$$
\varkappa_{n-1}^{K}(a)=a_{n-1},
$$

где $a_{n-1}$ - старшая координата элемента $a$ в координатном множестве $K$.

Пусть $u$ - последовательность элементов кольца $\mathbb{Z}_{2^{n}}$. Рассмотрим координатное множество $K$ кольца $\mathbb{Z}_{2^{n}}$ и последовательность $u_{n-1}$ над множеством $K$, полученную из последовательности $u$ по правилу

$$
u_{n-1}(i)=\varkappa_{n-1}^{K}(u(i)), i \geq 0 .
$$

Последовательность $u_{n-1}$ будем называть старшей координатной последовательностью последовательности $u$ в координатном множестве $K$ и в этом случае будем использовать обозначение $u_{n-1}=x_{n-1}^{K}(u)$.

Старшую координатную последовательность $u_{n-1}=\varkappa_{n-1}^{K}(u)$ часто рассматривают как усложнение исходной последовательности $u$. Это связано с тем, что, как правило, последовательность $u_{n-1}$ устроена достаточно сложно. Так, если $u$ - линейная рекуррентная последовательность (ЛРП) максимального периода над кольцом $\mathbb{Z}_{2^{n}}$, имеющая ранг $m$, то для произвольного координатного множества $K$ период последовательности $u_{n-1}$ совпадает с периодом последовательности $u$ (см., например, [1, теорема 1.3]). Если дополнительно считать, что $K=\left\{0, k_{1}\right\}$, то ранг последовательности $u_{n-1}$ 
будет равен $O\left(m^{2^{n-1}}\right)$ при $m \rightarrow \infty$ (см., например, [2, теорема 5.2]). Кроме того, если $K$ - двоичное координатное множество, то по последовательности $u_{n-1}$ однозначно восстанавливается вся исходная последовательность $u$ (см., например, [3, теорема 2]).

При исследовании частотных характеристик последовательностей $u_{n-1}=\varkappa_{n-1}^{K}(u)$ рассматривается двоичное координатное множество $K$. Представляет интерес исследование старших координатных последовательностей в произвольном координатном множестве $K$ с целью расширения класса преобразований усложнения для последовательности $u$. Другой причиной исследования произвольных множеств $K$ является то, что ранг последовательности $u_{n-1}=\varkappa_{n-1}^{K}(u)$ может зависеть от выбора $K$. В работе [4] этот эффект был подтвержден для случая, когда $n=2$. Еще один аргумент в пользу рассмотрения произвольного координатного множества $K$ связан с тем, что частоты появлений элементов в последовательности $u_{n-1}=\varkappa_{n-1}^{K}(u)$ при каждой фиксированной ЛРП $u$ также зависят от выбора $K$. В данной работе будут приведены соответствующие примеры для ЛРП над кольцом $\mathbb{Z}_{4}$.

Всюду в дальнейшем будем исследовать величину $N_{l}\left(z, u_{n-1}\right)$, равную количеству появлений элемента $z$ координатного множества $K$ среди первых $l$ элементов $u_{n-1}(0), u_{n-1}(1), \ldots, u_{n-1}(l-1)$ последовательности $u_{n-1}=\varkappa_{n-1}^{K}(u)$. Отметим, что имеет место равенство

$$
N_{l}\left(z, u_{n-1}\right)=\sum_{a \in \mathbb{Z}_{2^{n}}: \varkappa_{n-1}^{K}(a)=z} N_{l}(a, u)
$$

где $N_{l}(a, u)$ - количество элементов $a$ среди элементов $u(0), u(1), \ldots, u(l-1)$. Поэтому многие результаты о числах $N_{l}\left(z, u_{n-1}\right)$ получаются как следствия из описания частотных характеристик самой последовательности $u$, которые ранее были изучены (см., например, [5], [6]).

Представляет интерес нахождение других методов исследования частот $N_{l}\left(z, u_{n-1}\right)$, позволяющих получать более точные результаты. Один из таких подходов был предложен в работах [7], [8], [9], где в случае $K=\{0,1\}$ получены оценки частот появлений элементов на циклах старших координатных последовательностей линейных рекуррент максимального периода над кольцом $\mathbb{Z}_{2^{n}}$. Результаты этих работ улучшили ранее известные оценки рассматриваемых частот из [10] и [11]. Впоследствии этот подход был развит в работе [12], где получены оценки частот $N_{l}\left(z, u_{n-1}\right)$ при произвольных значениях $l$. В данной работе будет предложено дальнейшее развитие этого метода, с использованием которого доказываются более общие результаты: во-первых, изучены линейные рекурренты $u$, характеристический много- 
член которых, будучи приведенным по модулю два, является неприводимым многочленом над полем $G F(2)$ (условие максимальности периода при этом не требуется); во-вторых, исследуются старшие координатные последовательности $u_{n-1}=\varkappa_{n-1}^{K}(u)$ в произвольном координатном множестве $K$. Кроме того, доказывается, что полученные оценки являются асимптотически неулучшаемыми.

Заметим, что при исследовании частот $N_{l}\left(z, u_{n-1}\right)$ и связанных с ними частот $N_{l}(a, u)$ используются также другие подходы, отличные от метода тригонометрических сумм. Так, в работах [5] и [13] с использованием квадратичных форм найдены точные значения исследуемых частот на циклах ЛРП максимального периода и так называемых отмеченных ЛРП над кольцом $\mathbb{Z}_{4}$. В работе [14] комбинаторным методом получены точные значения частот $N_{l}(a, u)$ на циклах каждой ЛРП максимального периода над кольцом $\mathbb{Z}_{2^{n}}$, имеющей ранг, равный двум. Также с использованием комбинаторного подхода в работе [15] удалось оценить величину $N_{l}\left(0, u_{n-1}\right) / N_{l}\left(1, u_{n-1}\right)$, где $l$ равно периоду последовательности $u_{n-1}$, в терминах частоты $N_{l}(0, u)$ для каждой ЛРП $u$ максимального периода над кольцом $\mathbb{Z}_{2^{n}}$. Это позволило показать, что рассматриваемая дробь близка к единице практически для всех ЛРП максимального периода, имеющих заданный характеристический многочлен. В работе [16] вероятностными методами исследуется частота $N_{l}(a, u)$ при фиксированном характеристическом многочлене максимального периода над кольцом $\mathbb{Z}_{2^{n}}$ и случайном равновероятном выборе начального заполнения последовательности $u$. С использованием неравенства Чебышева получены оценки сверху вероятности $P\left(\left|N_{l}(a, u) / l-1 / 2^{n}\right| \geq \epsilon\right)$, где $\epsilon>0$. Все эти результаты обладают следующими недостатками: они либо являются довольно частными, либо не позволяют оценить частоты $N_{l}\left(z, u_{n-1}\right)$ для каждой конкретной последовательности $u$, так как описывают характеристики всего класса последовательностей с данным характеристическим многочленом. Таким образом, важное значение имеет развитие метода тригонометрических сумм для исследования рассматриваемых частот, который лишен указанных недостатков.

\section{2. Спектральный метод}

В этом параграфе будет развит спектральный метод, впервые предложенный в статье [7] и использовавшийся в работе [8] для описания частотных характеристик старшей двоичной координатной последовательности линейной рекурренты максимального периода над кольцом $\mathbb{Z}_{2^{n}}$. Этот метод позволяет свести исследование частоты $N_{l}\left(z, u_{n-1}\right)$ к исследованию соответству- 
ющих спектральных коэффициентов и тригонометрической суммы от ЛРП над кольцом $\mathbb{Z}_{2^{n}}$, что дает возможность применять уже известные оценки тригонометрических сумм над кольцами Галуа.

Укажем важное свойство отображения $\varkappa_{n-1}^{K}$.

Утверждение 1. Если $K=\left\{k_{0}, k_{1}\right\}$-координатное множество кольчза $\mathbb{Z}_{2^{n}, \text { mo }}$

1) $\varkappa_{n-1}^{K}(a)=k_{0}$ при всех $a \in\left\{k_{0}^{\prime}+k_{1}^{\prime} i: i=0,1, \ldots, 2^{n-1}-1\right\}$,

2) $\varkappa_{n-1}^{K}(a)=k_{1}$ при всех $a \in\left\{k_{0}^{\prime}+k_{1}^{\prime} i: i=2^{n-1}, 2^{n-1}+1, \ldots, 2^{n}-1\right\}$, где $k_{0}^{\prime}=-k_{0}, k_{1}^{\prime}=k_{1}-k_{0}$.

Доказательство. Заметим, что имеет место равенство $-k_{0}=k_{0}+2 k_{0}+$ $+2^{2} k_{0}+\ldots+2^{n-1} k_{0}$. Для каждого элемента $i$ из множества $\mathbb{Z}_{2^{n}}$ рассмотрим его двоичное представление $i=i_{0}+2 i_{1}+2^{2} i_{2}+\ldots+2^{n-1} i_{n-1}$, тогда получим равенство

$$
\begin{aligned}
-k_{0}+\left(k_{1}-k_{0}\right) i=\left(k_{0}+\ldots+2^{n-1} k_{0}\right)+ & \left(i_{0} k_{1}+\ldots+2^{n-1} i_{n-1} k_{1}\right)- \\
& -\left(i_{0} k_{0}+\ldots+2^{n-1} i_{n-1} k_{0}\right) .
\end{aligned}
$$

Заметим, что если $i_{s}=0$, то коэффициент при $2^{s}$ равен $k_{0}$, а если $i_{s}=1$, то коэффициент при $2^{s}$ равен $k_{1}$ для всех $s \in\{0,1, \ldots, n-1\}$. Таким образом, элемент $k_{0}^{\prime}+k_{1}^{\prime} i$ имеет старшую координату $k_{0}$ в координатном множестве $K$ тогда и только тогда, когда старшая двоичная координата $i$ равна 0 , и имеет старшую координату $k_{1}$ тогда и только тогда, когда старшая двоичная координата $i$ равна 1 .

Рассмотрим аддитивный характер $\chi$ кольца $\mathbb{Z}_{2^{n}}$, определенный на элементах этого кольца равенством

$$
\chi(x)=e^{2 \pi i \frac{x}{2^{n}}} .
$$

Утверждение 2. Пусть $K-$ координатное множество кольияа $\mathbb{Z}_{2^{n}}$. Тогда для всех $a \in \mathbb{Z}_{2^{n}}$ имеет место равенство

$$
(-1)^{\varkappa_{n-1}^{K}(a)}=\sum_{j=0}^{2^{n}-1} \mu_{j} \chi(a j),
$$

где

$$
\mu_{j}=\frac{1}{2^{n}} \sum_{s=0}^{2^{n}-1}(-1)^{\varkappa_{n-1}^{K}(s)} \chi(-s j) .
$$


Доказательство. С использованием соотношения ортогональности для характеров получим, что при всех $b \in \mathbb{Z}_{p^{n}}$

$$
\sum_{j=0}^{2^{n}-1} \chi(b j)=\left\{\begin{array}{cc}
0, & \text { если } b \neq 0, \\
2^{n}, & \text { если } b=0 .
\end{array}\right.
$$

Справедливо равенство

$$
\sum_{j=0}^{2^{n}-1} \mu_{j} \chi(a j)=\frac{1}{2^{n}} \sum_{j=0}^{2^{n}-1} \sum_{s=0}^{2^{n}-1}(-1)^{\varkappa_{n-1}^{K}(s)} \chi(-s j) \chi(a j) .
$$

Изменив в правой части этого равенства порядок суммирования и воспользовавшись равенствами (5), будем иметь

$$
\frac{1}{2^{n}} \sum_{s=0}^{2^{n}-1}(-1)^{\varkappa_{n-1}^{K}(s)} \sum_{j=0}^{2^{n}-1} \chi((a-s) j)=(-1)^{\varkappa_{n-1}^{K}(a)} .
$$

Величины $\mu_{i}$, участвующие в равенстве (3), можно рассматривать как соответствующие спектральные коэффициенты в представлении величины $(-1)^{\varkappa_{n-1}^{K}(a)}$ через характеры $\chi(a j)$, где $j=0,1, \ldots, 2^{n}-1$. Укажем свойства этих коэффициентов, которые понадобятся в дальнейшем.

Лемма 1. Если $K=\left\{k_{0}, k_{1}\right\}$ - координатное множество кольиа $\mathbb{Z}_{2^{n}}$, $k_{0}^{\prime}=-k_{0}, k_{1}^{\prime}=k_{1}-k_{0}$, mo

$$
\mu_{j}= \begin{cases}0, & \text { если } j-\text { четное число }, \\ \frac{1}{2^{n-1}} \sum_{i=0}^{2^{n-1}-1} \chi\left(-\left(k_{0}^{\prime}+k_{1}^{\prime} i\right) j\right), & \text { если } j-\text { нечетное число }\end{cases}
$$

Доказательство. Из утверждения 1 следует, что

$$
(-1)^{\varkappa_{n-1}^{K}(s)}=\left\{\begin{aligned}
1, & \text { если } s \in\left\{k_{0}^{\prime}+k_{1}^{\prime} i: i=0,1, \ldots, 2^{n-1}-1\right\}, \\
-1, & \text { если } s \in\left\{k_{0}^{\prime}+k_{1}^{\prime} i: i=2^{n-1}, 2^{n-1}+1, \ldots, 2^{n}-1\right\} .
\end{aligned}\right.
$$

Тогда в силу равенства (4) справедливы соотношения

$$
\begin{aligned}
\mu_{j}=\frac{1}{2^{n}} \sum_{s=0}^{2^{n}-1}(-1)^{\varkappa_{n-1}^{K}}(s) & \chi(-s j)= \\
& =\frac{1}{2^{n}} \sum_{i=0}^{2^{n-1}-1} \chi\left(-\left(k_{0}^{\prime}+k_{1}^{\prime} i\right) j\right)-\frac{1}{2^{n}} \sum_{i=2^{n-1}}^{2^{n}-1} \chi\left(-\left(k_{0}^{\prime}+k_{1}^{\prime} i\right) j\right),
\end{aligned}
$$


а значит,

$$
\mu_{j}=\frac{1}{2^{n}} \sum_{i=0}^{2^{n-1}-1}\left(\chi\left(-\left(k_{0}^{\prime}+k_{1}^{\prime} i\right) j\right)-\chi\left(-\left(k_{0}^{\prime}+k_{1}^{\prime} i\right) j\right) \chi\left(-k_{1}^{\prime} 2^{n-1} j\right)\right) .
$$

В силу того, что число $k_{1}^{\prime}$ нечетно, выполняется равенство $\chi\left(-k_{1}^{\prime} 2^{n-1} j\right)=$ $=(-1)^{j}$. Таким образом, если $j-$ четное число, то $\mu_{j}=0$, а если $j-$ нечетное, то

$$
\mu_{j}=\frac{1}{2^{n-1}} \sum_{i=0}^{2^{n-1}-1} \chi\left(-\left(k_{0}^{\prime}+k_{1}^{\prime} i\right) j\right)
$$

Основным результатом этого параграфа является следующая оценка суммы модулей всех спектральных коэффициентов $\mu_{j}$.

Теорема 1. При всех $n \in \mathbb{N}$ имеет место оценка

$$
\sum_{j=0}^{2^{n}-1}\left|\mu_{j}\right| \leq \frac{2}{\pi} \ln \left(2^{n-1}\right)+1
$$

которая только при $n=1$ обращуается в равенство.

Доказательство. Если $n=1$, то доказываемое неравенство очевидно. Пусть теперь $n \geq 2$. Согласно лемме 1

$$
\sum_{j=0}^{2^{n}-1}\left|\mu_{j}\right|=\sum_{s=0}^{2^{n-1}-1}\left|\mu_{2 s+1}\right|=\frac{1}{2^{n-1}} \sum_{s=0}^{2^{n-1}-1}\left|\chi\left(-k_{0}^{\prime}(2 s+1)\right) \sum_{t=0}^{2^{n-1}-1} \chi\left(-k_{1}^{\prime} t(2 s+1)\right)\right|
$$

Так как $\left|\chi\left(-k_{0}^{\prime}(2 s+1)\right)\right|=1$ и нечетные числа $k_{1}^{\prime}(2 s+1)$, где $s=$ $=0,1, \ldots, 2^{n-1}-1$, попарно не сравнимы по модулю $2^{n}$, то справедливы равенства

$$
\begin{aligned}
& \sum_{j=0}^{2^{n}-1}\left|\mu_{j}\right|=\frac{1}{2^{n-1}} \sum_{s=0}^{2^{n-1}-1}\left|\sum_{t=0}^{2^{n-1}-1} \chi\left(-k_{1}^{\prime} t(2 s+1)\right)\right|= \\
&=\frac{1}{2^{n-1}} \sum_{s=0}^{2^{n-1}-1}\left|\sum_{t=0}^{2^{n-1}-1} \chi((2 s+1) t)\right| .
\end{aligned}
$$

2010, T. 1, № 4, C. 33-62 
Тогда, учитывая равенство (2), будем иметь

$$
\sum_{j=0}^{2^{n}-1}\left|\mu_{j}\right|=\frac{1}{2^{n-1}} \sum_{s=0}^{2^{n-1}-1}\left|\sum_{t=0}^{2^{n-1}-1} e^{2 \pi i \frac{2 s+1}{2^{n}} t}\right|=\frac{1}{2^{n-1}} \sum_{s=0}^{2^{n-1}-1}\left|\frac{e^{\pi i(2 s+1)}-1}{e^{2 \pi i \frac{2 s+1}{2^{n}}}-1}\right| .
$$

В итоге получим

$$
\sum_{j=0}^{2^{n}-1}\left|\mu_{j}\right|=\frac{1}{2^{n-1}} \sum_{s=0}^{2^{n-1}-1} \frac{1}{\sin \left(\frac{2 s+1}{2^{n}} \pi\right)}=\frac{1}{2^{n-2}} \sum_{s=0}^{2^{n-2}-1} \frac{1}{\sin \left(\frac{2 s+1}{2^{n}} \pi\right)}
$$

При $n=2$ правая часть полученного равенства равна $\sqrt{2}=1,41 \ldots$, что меньше, чем $(2 / \pi) \ln 2+1=1,44 \ldots$. При $n=3$ правая часть рассматриваемого равенства равна $(1 / 2)\left((\sin (\pi / 8))^{-1}+(\sin (3 \pi / 8))^{-1}\right)=1,84 \ldots$, что меньше, чем $(2 / \pi) \ln 4+1=1,88 \ldots$ Поэтому будем исследовать случай, когда $n \geq 4$. Согласно интегральному неравенству Йенсена (см., например, $[17$, стр. 153]) для выпуклой и дифференцируемой функции $f(x)$ на отрезке $[a, b]$ имеет место неравенство

$$
f\left(\frac{a+b}{2}\right) \leq \frac{1}{b-a} \int_{a}^{b} f(x) d x
$$

Применив это неравенство для $f(x)=1 / \sin x$ на отрезках $\left[\pi(j-1) / 2^{n}, \pi(j+\right.$ $\left.+1) / 2^{n}\right]$, где $j=3,5, \ldots, 2^{n-1}-1$, будем иметь

$$
\sum_{j=0}^{2^{n}-1}\left|\mu_{j}\right| \leq \frac{1}{2^{n-2} \sin \left(\frac{\pi}{2^{n}}\right)}+\frac{1}{2^{n-2}} \sum_{j=3,5, \ldots, 2^{n-1}-1} \frac{2^{n-1}}{\pi} \int_{\frac{\pi(j-1)}{2^{n}}}^{\frac{\pi(j+1)}{2^{n}}} \frac{1}{\sin x} d x,
$$

а тогда

$$
\begin{aligned}
\sum_{j=0}^{2^{n}-1}\left|\mu_{j}\right| \leq \frac{1}{2^{n-2} \sin \left(\frac{\pi}{2^{n}}\right)} & +\frac{2}{\pi} \int_{\frac{\pi}{2^{n-1}}}^{\frac{\pi}{2}} \frac{1}{\sin x} d x= \\
= & \frac{1}{2^{n-2} \sin \left(\frac{\pi}{2^{n}}\right)}+\frac{2}{\pi}\left(\ln \left(\operatorname{tg}\left(\frac{\pi}{4}\right)\right)-\ln \left(\operatorname{tg}\left(\frac{\pi}{2^{n}}\right)\right)\right) .
\end{aligned}
$$

Так как $\operatorname{tg} x>x$ при всех $x \in\left(0, \frac{\pi}{4}\right)$, то

$$
\sum_{j=0}^{2^{n}-1}\left|\mu_{j}\right| \leq \frac{1}{2^{n-2} \sin \left(\frac{\pi}{2^{n}}\right)}-\frac{2}{\pi} \ln \left(\operatorname{tg}\left(\frac{\pi}{2^{n}}\right)\right)<\frac{1}{2^{n-2} \sin \left(\frac{\pi}{2^{n}}\right)}+\frac{2}{\pi} \ln \left(\frac{2^{n}}{\pi}\right) .
$$


Функция $(\sin x) / x$ убывает на интервале $\left(0, \frac{\pi}{4}\right)$, поэтому при $n \geq 4$ справедливо неравенство

а значит,

$$
\frac{\sin \left(\frac{\pi}{2^{n}}\right)}{\frac{\pi}{2^{n}}} \geq \frac{\sin \left(\frac{\pi}{16}\right)}{\frac{\pi}{16}}
$$

$$
\frac{1}{2^{n-2} \sin \left(\frac{\pi}{2^{n}}\right)} \leq \frac{\frac{\pi}{16}}{2^{n-2} \frac{\pi}{2^{n}} \sin \left(\frac{\pi}{16}\right)}=\frac{1}{4 \sin \left(\frac{\pi}{16}\right)}=1,281 \ldots
$$

Учитывая, что $(2 / \pi) \ln (\pi / 2)=0,287 \ldots$, получим

$$
\sum_{j=0}^{2^{n}-1}\left|\mu_{j}\right|<\frac{2}{\pi} \ln \left(2^{n-1}\right)+\frac{1}{2^{n-2} \sin \left(\frac{\pi}{2^{n}}\right)}-\frac{2}{\pi} \ln \left(\frac{\pi}{2}\right)<\frac{2}{\pi} \ln \left(2^{n-1}\right)+1 .
$$

Ранее в работе [7, следствие 14] для двоичного координатного множества была получена оценка

$$
\sum_{j=0}^{2^{n}-1}\left|\mu_{j}\right|<\frac{2}{\pi} \ln \left(2^{n}\right)+1
$$

Правая часть этого неравенства больше правой части неравенства из теоремы 1 на величину $(2 / \pi) \ln 2=0,441 \ldots$

\section{3. Распределение элементов на циклах координатных последовательностей}

Пусть $F(x)$ - унитарный многочлен степени $m$ над кольцом $R=\mathbb{Z}_{2^{n}}$. Назовем многочлен $F(x)$ реверсивным многочленом Галуа над кольцом $R$, если многочлен $\bar{F}(x)$, полученный из $F(x)$ приведением всех его коэффициентов по модулю 2 , является неприводимым многочленом над полем $G F(2)$ и $\bar{F}(x) \neq x$. Обозначим через $L_{R}(F)$ множество всех ЛРП $u$ над кольцом $R$ с характеристическим многочленом $F(x)$, а через $L_{R}(F)^{*}-$ множество всех таких ЛРП $u$, у которых в начальном отрезке $(u(0), u(1), \ldots, u(m-1))$ есть хотя бы один обратимый элемент кольца $R$. Нулевую последовательность будем обозначать (0). Если $F(x)$ - реверсивный многочлен Галуа над кольцом $R$, то каждая последовательность $u \in L_{R}(F)^{*}$ будет чисто периодической последовательностью периода $T(u)$, который совпадает с периодом $T(F)$ многочлена $F(x)$ (см., например, [2]).

Следующее утверждение позволяет перейти от исследования частоты $N_{l}\left(z, u_{n-1}\right)$ к изучению некоторой суммы степеней числа -1. 
Утверждение 3. Пусть $K=\left\{k_{0}, k_{1}\right\}$ - координатное множество кольиа $\mathbb{Z}_{2^{n}}, u-$ последовательность над кольцом $\mathbb{Z}_{2^{n}}, u_{n-1}=\varkappa_{n-1}^{K}(u), l \in \mathbb{N}$, $z \in K$, тогда

$$
N_{l}\left(z, u_{n-1}\right)=\frac{l}{2}+\frac{(-1)^{z}}{2} \sum_{i=0}^{l-1}(-1)^{\varkappa_{n-1}^{K}(u(i))} .
$$

Доказательство. Заметим, что при всех $x \in \mathbb{Z}$ имеет место соотношение

$$
\frac{1}{2}\left(1+(-1)^{x}\right)= \begin{cases}1, & \text { если } x-\text { четное число, } \\ 0, & \text { если } x-\text { нечетное число. }\end{cases}
$$

С использованием этого равенства получим

$$
N_{l}\left(z, u_{n-1}\right)=\sum_{i=0}^{l-1} \frac{1}{2}\left(1+(-1)^{u_{n-1}(i)+z}\right)=\frac{l}{2}+\frac{(-1)^{z}}{2} \sum_{i=0}^{l-1}(-1)^{\varkappa_{n-1}^{K}(u(i))} .
$$

Лемма 2. ([6]). Если $F(x)$ - реверсивный многочлен Галуа степени $m$ над кольцом $R=\mathbb{Z}_{2^{n}}, T(F)=2^{\nu}\left(2^{m}-1\right) / d, 0 \leq \nu \leq n-1, v \in L_{R}(F), v \neq(0)$, $\chi$ - аддитивный характер кольциа $R$, определенный равенством (2), то

$$
\left|\sum_{i=0}^{T(F)-1} \chi(v(i))+\frac{2^{\nu}}{d}\right| \leq \frac{2^{\nu}\left(d 2^{n-1}-1\right)}{d} 2^{\frac{m}{2}} .
$$

Лемма 3. Для чисел $\mu_{j}$, определенных формулой (4), справедливо равенство

$$
\sum_{j=0}^{2^{n}-1} \mu_{j}=(-1)^{\varkappa_{n-1}^{K}(0)}
$$

Доказательство. Используя соотношение ортогональности (5), получим

$$
\begin{aligned}
& \sum_{j=0}^{2^{n}-1} \mu_{j}=\sum_{j=0}^{2^{n}-1} \frac{1}{2^{n}} \sum_{s=0}^{2^{n}-1}(-1)^{\varkappa_{n-1}^{K}(s)} \chi(-s j)= \\
&=\frac{1}{2^{n}} \sum_{s=0}^{2^{n}-1}(-1)^{\varkappa_{n-1}^{K}(s)} \sum_{j=0}^{2^{n}-1} \chi(-s j)=(-1)^{\varkappa_{n-1}^{K}(0)} .
\end{aligned}
$$

Докажем основной результат этого параграфа. 
Теорема 2. Пусть $F(x)$ - реверсивный многочлен Галуа степени $m$ над кольцом $R=\mathbb{Z}_{2^{n}}, n \geq 2, T(F)=2^{\nu}\left(2^{m}-1\right) / d, 0 \leq \nu \leq n-1, u \in L_{R}(F)^{*}$, $K-$ координатное множество кольиа $\mathbb{Z}_{2^{n}}, u_{n-1}=\varkappa_{n-1}^{K}(u), z \in K$. Тогда для числа $N\left(z, u_{n-1}\right)=N_{T(F)}\left(z, u_{n-1}\right)$ справедлива оченка

$$
\left|N\left(z, u_{n-1}\right)-\omega(z)\right|<\left(\frac{1}{\pi} \ln \left(2^{n-1}\right)+\frac{1}{2}\right) \frac{2^{\nu}\left(d 2^{n-1}-1\right)}{d} 2^{\frac{m}{2}},
$$

где

$$
\omega(z)= \begin{cases}\frac{2^{m-1}-1}{2^{m}-1} T(F), & \text { если } z=\varkappa_{n-1}^{K}(0), \\ \frac{2^{m-1}}{2^{m}-1} T(F), & \text { если } z \neq \varkappa_{n-1}^{K}(0) .\end{cases}
$$

Доказательство. Согласно утверждению 3

$$
N\left(z, u_{n-1}\right)-\frac{T(F)}{2}=\frac{(-1)^{z}}{2} \sum_{i=0}^{T(F)-1}(-1)^{\varkappa_{n-1}^{K}(u(i))} .
$$

Отсюда и из равенства (3) следует, что

$$
N\left(z, u_{n-1}\right)-\frac{T(F)}{2}=\frac{(-1)^{z}}{2} \sum_{i=0}^{T(F)-1} \sum_{j=0}^{2^{n}-1} \mu_{j} \chi(u(i) j) .
$$

Изменив порядок суммирования и добавив во внутреннюю сумму слагаемое $2^{\nu} / d$, получим

$$
N\left(z, u_{n-1}\right)-\frac{T(F)}{2}=\frac{(-1)^{z}}{2} \sum_{j=0}^{2^{n}-1} \mu_{j} \sum_{i=0}^{T(F)-1}\left(\chi(u(i) j)+\frac{2^{\nu}}{d}\right)-\frac{(-1)^{z}}{2} \sum_{j=0}^{2^{n}-1} \mu_{j} \frac{2^{\nu}}{d} .
$$

С использованием леммы 3 имеем

$$
\frac{T(F)}{2}-\frac{(-1)^{z}}{2} \sum_{j=0}^{2^{n}-1} \mu_{j} \frac{2^{\nu}}{d}=\frac{T(F)}{2}-\frac{2^{\nu}(-1)^{z+\varkappa_{n-1}^{K}(0)}}{2 d}=\omega(z) .
$$

Так как $\mu_{0}=0$, то справедлива оценка

$$
\left|N\left(z, u_{n-1}\right)-\omega(z)\right| \leq \frac{1}{2}\left(\sum_{j=0}^{2^{n}-1}\left|\mu_{j}\right|\right) \max _{v \in L_{R}(F), v \neq(0)}\left|\sum_{i=0}^{T(F)-1}\left(\chi(v(i))+\frac{2^{\nu}}{d}\right)\right| .
$$

2010, T. 1, № 4, C. 33-62 
Для завершения доказательства остается воспользоваться теоремой 1 и леммой 2.

Ранее в [9, теорема 4] было показано, что в условиях теоремы 2 при $n \geq 4, d=1, \nu=n-1, K=\{0,1\}$ справедлива оценка

$$
\left|N\left(z, u_{n-1}\right)-\frac{T(F)}{2}\right|<\left(\frac{1}{\pi} \ln \left(2^{n}\right)+\frac{1}{2}\right) 2^{n-1}\left(\left(2^{n-1}-1\right) 2^{\frac{m}{2}}+1\right) .
$$

Обозначим через $S(F)$ правую часть неравенства (7), тогда справедливы оценки

$$
\frac{T(F)}{2}-S(F)<N\left(z, u_{n-1}\right)<\frac{T(F)}{2}+S(F) .
$$

Нетрудно заметить, что если даже в указанных нижней и верхней оценках частоты $N\left(z, u_{n-1}\right)$ заменить величину $(1 / \pi) \ln \left(2^{n}\right)+1 / 2$ на меньшую $(1 / \pi) \ln \left(2^{n-1}\right)+1 / 2$, то они оказываются хуже, чем аналогичные оценки, вытекающие из теоремы 2.

\section{4. Отмеченные ЛРП над кольцом $\mathbb{Z}_{4}$}

В этом параграфе рассмотрим кольцо вычетов $R=\mathbb{Z}_{4}$. Реверсивный многочлен Галуа $F(x)$ степени $m$ над кольцом $R$, имеющий период $T(F)=T(\bar{F})=2^{m}-1$, будем называть отмеченным многочленом. Период отмеченного многочлена в два раза меньше максимально возможного периода $2\left(2^{m}-1\right)$ для многочленов степени $m$ над кольцом $R$. Каждую ЛРП $u$ из множества $L_{R}(F)^{*}$ с отмеченным характеристическим многочленом $F(x)$ будем называть отмеченной. Для отмеченной ЛРП $u$ выполнено равенство $T(u)=T(F)=2^{m}-1$. Вопрос о числе $N(z, u)$ появлений элемента $z \in \mathbb{Z}_{4}$ среди элементов $u(0), u(1), \ldots, u(T(u)-1)$ отмеченной ЛРП $u$ решен в работе [18] и в более общем случае - в [13]. Приведем результаты этих работ, из которых будут следовать точные значения для количества появлений элементов в старших координатных последовательностях $u_{1}=\varkappa_{1}^{K}(u)$ (для координатных множеств $K=\left\{k_{0}, k_{1}\right\}$ ).

Назовем типом распределения элементов в последовательности $u$ упорядоченный набор $(N(0, u), N(1, u), N(2, u), N(3, u))$, а кратностью типа назовем количество последовательностей из множества $L_{R}(F)^{*}$, имеющих данный тип распределения.

Теорема 3. ([13], [18]). Пусть $F(x)$ - отмеченный многочлен степени т над кольцом $R=\mathbb{Z}_{4}$, тогда для отмеченных ЛРП $и \in L_{R}(F)^{*}$ возможны четыре типа распределений, указанные в следующих таблицах: 
1) $п$ пи $m=2 \lambda$

\begin{tabular}{|c|c|c|c|c|}
\hline$N(0, u)$ & $N(1, u)$ & $N(2, u)$ & $N(3, u)$ & $\begin{array}{c}\text { кратность } \\
\text { типа }\end{array}$ \\
\hline $2^{m-2}+2^{\lambda-1}-1$ & $2^{m-2}$ & $2^{m-2}-2^{\lambda-1}$ & $2^{m-2}$ & $\begin{array}{c}\left(2^{m-2}+2^{\lambda-1}\right) \times \\
\times\left(2^{m}-1\right)\end{array}$ \\
\hline $2^{m-2}-2^{\lambda-1}-1$ & $2^{m-2}$ & $2^{m-2}+2^{\lambda-1}$ & $2^{m-2}$ & $\begin{array}{c}\left(2^{m-2}-2^{\lambda-1}\right) \times \\
\times\left(2^{m}-1\right)\end{array}$ \\
\hline $2^{m-2}-1$ & $2^{m-2}+2^{\lambda-1}$ & $2^{m-2}$ & $2^{m-2}-2^{\lambda-1}$ & $2^{m-2}\left(2^{m}-1\right)$ \\
\hline $2^{m-2}-1$ & $2^{m-2}-2^{\lambda-1}$ & $2^{m-2}$ & $2^{m-2}+2^{\lambda-1}$ & $2^{m-2}\left(2^{m}-1\right)$ \\
\hline
\end{tabular}

2) $п р и т=2 \lambda+1$

\begin{tabular}{|c|c|c|c|c|}
\hline$N(0, u)$ & $N(1, u)$ & $N(2, u)$ & $N(3, u)$ & $\begin{array}{c}\text { кратность } \\
\text { типа }\end{array}$ \\
\hline $2^{m-2}+2^{\lambda-1}-1$ & $2^{m-2}+2^{\lambda-1}$ & $2^{m-2}-2^{\lambda-1}$ & $2^{m-2}-2^{\lambda-1}$ & $\begin{array}{c}\left(2^{m-2}+2^{\lambda-1}\right) \times \\
\times\left(2^{m}-1\right)\end{array}$ \\
\hline $2^{m-2}+2^{\lambda-1}-1$ & $2^{m-2}-2^{\lambda-1}$ & $2^{m-2}-2^{\lambda-1}$ & $2^{m-2}+2^{\lambda-1}$ & $\begin{array}{c}\left(2^{m-2}+2^{\lambda-1}\right) \times \\
\times\left(2^{m}-1\right)\end{array}$ \\
\hline $2^{m-2}-2^{\lambda-1}-1$ & $2^{m-2}+2^{\lambda-1}$ & $2^{m-2}+2^{\lambda-1}$ & $2^{m-2}-2^{\lambda-1}$ & $\begin{array}{c}\left(2^{m-2}-2^{\lambda-1}\right) \times \\
\times\left(2^{m}-1\right)\end{array}$ \\
\hline $2^{m-2}-2^{\lambda-1}-1$ & $2^{m-2}-2^{\lambda-1}$ & $2^{m-2}+2^{\lambda-1}$ & $2^{m-2}+2^{\lambda-1}$ & $\begin{array}{c}\left(2^{m-2}-2^{\lambda-1}\right) \times \\
\times\left(2^{m}-1\right)\end{array}$ \\
\hline
\end{tabular}

Пусть $K=\left\{k_{0}, k_{1}\right\}$ - координатное множество кольца $R=\mathbb{Z}_{4}, F(x)$ отмеченный многочлен над кольцом $R, u \in L_{R}(F)^{*}, u_{1}=\varkappa_{1}^{K}(u)-$ старшая координатная последовательность ЛРП $u$. В качестве следствия из теоремы 3 получим число $N\left(z, u_{1}\right)$ появлений элемента $z \in K$ среди элементов $u_{1}(0), u_{1}(1), \ldots, u_{1}(T(u)-1)$ старшей координатной последовательности $u_{1}$ отмеченной ЛРП $u$. Назовем типом распределения элементов в последовательности $u_{1}$ упорядоченный набор $\left(N\left(k_{0}, u_{1}\right), N\left(k_{1}, u_{1}\right)\right)$, а кратностью типа назовем количество последовательностей $u$ из множества $L_{R}(F)^{*}$, у которых последовательность $u_{1}=\varkappa_{1}^{K}(u)$ имеет данный тип распределения.

Следствие 1. Пусть в условиях теоремь $3 K=\left\{k_{0}, k_{1}\right\}-$ координатное множество кольиа $R=\mathbb{Z}_{4}, u_{1}=\varkappa_{1}^{K}(u)$, тогда:

1) если $m=2 \lambda$, то при каждом $K$ возможны два типа распределений элементов в последовательности $u_{1}$, представленные в следующей таблице: 


\begin{tabular}{|c|c|c|c|}
\hline $\begin{array}{l}\text { Множество } \\
K=\left\{k_{0}, k_{1}\right\}\end{array}$ & $N\left(k_{0}, u_{1}\right)$ & $N\left(k_{1}, u_{1}\right)$ & кратность типа \\
\hline $\begin{array}{l}K=\{0,1\} \\
K=\{0,3\}\end{array}$ & $2^{m-1}+2^{\lambda-1}-1$ & $2^{m-1}-2^{\lambda-1}$ & $\left(2^{m-1}+2^{\lambda-1}\right)\left(2^{m}-1\right)$ \\
\hline $\begin{array}{l}K=\{0,1\} \\
K=\{0,3\}\end{array}$ & $2^{m-1}-2^{\lambda-1}-1$ & $2^{m-1}+2^{\lambda-1}$ & $\left(2^{m-1}-2^{\lambda-1}\right)\left(2^{m}-1\right)$ \\
\hline $\begin{array}{l}K=\{2,1\} \\
K=\{2,3\}\end{array}$ & $2^{m-1}-2^{\lambda-1}$ & $2^{m-1}+2^{\lambda-1}-1$ & $\left(2^{m-1}+2^{\lambda-1}\right)\left(2^{m}-1\right)$ \\
\hline $\begin{array}{l}K=\{2,1\} \\
K=\{2,3\}\end{array}$ & $2^{m-1}+2^{\lambda-1}$ & $2^{m-1}-2^{\lambda-1}-1$ & $\left(2^{m-1}-2^{\lambda-1}\right)\left(2^{m}-1\right)$ \\
\hline
\end{tabular}

2) если $m=2 \lambda+1$, то при каждом К возможны три типа распределений элементов в последовательности $u_{1}$, представленные в следующей таблице:

\begin{tabular}{|l|c|c|c|}
\hline Множество & $N\left(k_{0}, u_{1}\right)$ & $N\left(k_{1}, u_{1}\right)$ & кратность типа \\
$K=\left\{k_{0}, k_{1}\right\}$ & $2^{m-1}-1$ & $2^{m-1}$ & $2^{m-1}\left(2^{m}-1\right)$ \\
\hline $\begin{array}{l}K=\{0,1\}, \\
K=\{0,3\}\end{array}$ & $2^{m-1}-2^{\lambda}$ & $\left(2^{m-2}+2^{\lambda-1}\right)\left(2^{m}-1\right)$ \\
\hline $\begin{array}{l}K=\{0,1\}, \\
K=\{0,3\}\end{array}$ & $2^{m-1}+2^{\lambda}-1$ & $2^{m}$ & $\left(2^{m-2}-2^{\lambda-1}\right)\left(2^{m}-1\right)$ \\
\hline $\begin{array}{l}K=\{0,1\}, \\
K=\{0,3\}\end{array}$ & $2^{m-1}-2^{\lambda}-1$ & $2^{m-1}+2^{\lambda}$ & $2^{m-1}\left(2^{m}-1\right)$ \\
\hline $\begin{array}{l}K=\{2,1\}, \\
K=\{2,3\}\end{array}$ & $2^{m-1}$ & $2^{m-1}-1$ & $\left(2^{m-2}-2^{\lambda-1}\right)\left(2^{m}-1\right)$ \\
\hline $\begin{array}{l}K=\{2,1\}, \\
K=\{2,3\}\end{array}$ & $2^{m-1}+2^{\lambda}$ & $2^{m-1}-2^{\lambda}-1$ & $\left(2^{m-1}+2^{\lambda-1}\right)\left(2^{m}-1\right)$ \\
\hline $\begin{array}{l}K=\{2,1\}, \\
K=\{2,3\}\end{array}$ & $2^{m-1}-2^{\lambda}$ & $2^{m-1}+2^{\lambda}-1$ & \multicolumn{2}{|l}{} \\
\hline
\end{tabular}

Доказательство. Достаточно воспользоваться теоремой 3 и заметить, что для всех $z \in K$ имеет место равенство $N\left(z, u_{1}\right)=N(a, u)+N(b, u)$, где $a$, $b-$ элементы кольца $\mathbb{Z}_{4}$ такие, что $\varkappa_{1}^{K}(a)=\varkappa_{1}^{K}(b)=z$.

Из теоремы 3 следует, что каждая из отмеченных ЛРП $u$ над кольцом $R=\mathbb{Z}_{4}$ имеет «неидеальные» частотные характеристики. Из следствия 1 вытекает, что при всех четных $m$ и при всех координатных множествах $K$ частоты появлений элементов в последовательностях $u_{1}=\varkappa_{1}^{K}(u)$ также имеют существенные отклонения от среднего значения $2^{m-1}$. Однако при любом нечетном значении $m$ для каждого координатного множества $K$ найдутся отмеченные ЛРП $u$, у которых координатная последовательность $u_{1}=\varkappa_{1}^{K}(u)$ 
имеет тип распределения $\left(2^{m-1}-1,2^{m-1}\right)$ или $\left(2^{m-1}, 2^{m-1}-1\right)$. Причем ровно половина всех последовательностей $u \in L_{R}(F)^{*}$ с данным фиксированным отмеченным характеристическим многочленом $F(x)$ обладает этим свойством.

Кроме того, с помощью теоремы 3 несложно показать, что при нечетном $m$ для каждой ЛРП $u \in L_{R}(F)^{*}$ с отмеченным характеристическим многочленом $F(x)$ степени $m$ всегда найдется такое координатное множество $K$, что тип распределения последовательности $u_{1}=\varkappa_{1}^{K}(u)$ имеет вид $\left(2^{m-1}-1,2^{m-1}\right)$, а также найдется такое координатное множество $K$, что тип распределения последовательности $u_{1}=\varkappa_{1}^{K}(u)$ имеет вид $\left(2^{m-1}, 2^{m-1}-1\right)$. Таким образом, подбирая нужным образом координатное множество, можно на цикле старшей координатной последовательности $u_{1}=\varkappa_{1}^{K}(u)$ уменьшить отклонения частот появлений от среднего значения по сравнению с исходной последовательностью $u$.

Рассмотрим вопрос о том, насколько точной является оценка из теоремы 2 применительно к отмеченным ЛРП $u$ над кольцом $\mathbb{Z}_{4}$. Согласно теореме 2 для всех $z \in K$ имеет место неравенство

$2^{m-1}-\left(\frac{1}{\pi} \ln 2+\frac{1}{2}\right) 2^{\frac{m}{2}}-\delta(z)<N\left(z, u_{1}\right)<2^{m-1}+\left(\frac{1}{\pi} \ln 2+\frac{1}{2}\right) 2^{\frac{m}{2}}-\delta(z)$,

где $\delta(z)=0$, если $z \neq \varkappa_{1}^{K}(0)$, и $\delta(z)=1$, если $z=\varkappa_{1}^{K}(0)$. Таким образом, при $m=2 \lambda$ оценка из теоремы 2 устанавливает неравенства

$$
2^{m-1}-1,441 \cdot 2^{\lambda-1}-\delta(z)<N\left(z, u_{1}\right)<2^{m-1}+1,442 \cdot 2^{\lambda-1}-\delta(z),
$$

а при $m=2 \lambda+1$ оценка из теоремы 2 приводит к соотношениям

$$
2^{m-1}-1,019 \cdot 2^{\lambda}-\delta(z)<N\left(z, u_{1}\right)<2^{m-1}+1,02 \cdot 2^{\lambda}-\delta(z) .
$$

Сравнение этих результатов с таблицами из следствия 1 показывает, что для отмеченных ЛРП над кольцом $\mathbb{Z}_{4}$ они являются весьма точными.

\section{5. Распределение элементов на отрезках координат- ных последовательностей}

Рассмотрим вопрос о числе появлений элементов на отрезках координатных последовательностей. Нам понадобится несколько вспомогательных утверждений. 
Лемма 4 ([19]). $\quad$ Если $t-$ нечетное натуральное число, $l \in \mathbb{N}, l \leq t$, mo

$$
\frac{1}{t} \sum_{a=0}^{t-1}\left|\sum_{k=0}^{l-1} e^{2 \pi i \frac{-a k}{t}}\right|<\frac{2}{\pi} \ln \left(\frac{4 t}{\pi}\right)+1 .
$$

Отметим, что в формулировке этого результата (см. [19, лемма 1]) указано, что $t=2^{n}-1$. Однако на самом деле при доказательстве это равенство не используется, используется лишь условие, что $t$ - нечетное число.

Лемма 5. Пусть $F(x)$ - реверсивный многочлен Галуа степени $m$ над кольиом $R=\mathbb{Z}_{2^{n}}, T(F)=T(\bar{F})=t, v \in L_{R}(F), v \neq(0), l \in \mathbb{N}, \chi-$ характер кольиза $R$, определенный равенством (2). Тогда для всех $l \leq T(F)$ выполнено неравенство

$$
\left|\sum_{i=0}^{l-1} \chi(v(i))\right| \leq\left(\frac{2}{\pi} \ln \left(\frac{4 t}{\pi}\right)+1\right) 2^{\frac{m}{2}+n-1} .
$$

Доказательство. Достаточно повторить доказательство из [6, теорема 1] и воспользоваться оценкой из леммы 4.

Лемма 6. Пусть $F(x)$ - реверсивный многочлен Галуа степени $m$ над кольцом $R=\mathbb{Z}_{2^{n}}, T(F)=2^{\nu} T(\bar{F}), 0 \leq \nu \leq n-1, t=T(\bar{F}), v \in L_{R}(F)$, $v \neq(0), l \in \mathbb{N}, \chi-$ характер кольиа $R$, определенный равенством (2). Тогда для всех $l \leq T(F)$ выполнено неравенство

$$
\left|\sum_{i=0}^{l-1} \chi(v(i))\right| \leq\left(\frac{2}{\pi} \ln \left(\frac{4 t}{\pi}\right)+1\right) 2^{\frac{m}{2}+\nu+n-1} .
$$

Доказательство. Рассмотрим последовательности $v_{s}$ над кольцом $\mathbb{Z}_{2^{n}}$, определенные для всех $s=0,1, \ldots, 2^{\nu}-1$ равенством

$$
v_{s}(i)=u\left(s+2^{\nu} i\right), i \geq 0 .
$$

Пусть представление ЛРП v с использованием функции след (см., например, [2]) имеет вид

$$
v(i)=\operatorname{Tr}_{R}^{S}\left(b \alpha^{i}\right),
$$

где $S=G R\left(2^{m n}, 2^{n}\right)$ - кольцо Галуа, являющееся расширением степени $m$ кольца $R, \operatorname{Tr}_{R}^{S}-$ функция след из кольца $S$ в кольцо $R, \alpha$ - корень многочлена $F(x)$ в кольце $S, b-$ ненулевой элемент кольца $S$. Так как $F(x)-$ 
реверсивный многочлен Галуа, то $\alpha$ является обратимым элементом кольца $S$, причем порядок элемента $\alpha$ будет равен $T(F)$. Представление каждой ЛРП $v_{s}$ с использованием функции след будет следующим:

$$
v_{S}(i)=v\left(s+2^{\nu} i\right)=\operatorname{Tr}_{R}^{S}\left(b \alpha^{s} \alpha_{0}^{i}\right)
$$

где $\alpha_{0}=\alpha^{2^{\nu}}$ - элемент порядка $t=T(\bar{F})$. Элемент $\alpha_{0}-$ корень некоторого реверсивного многочлена Галуа $G(x)$ степени $m$ над кольцом $R$, причем $T(G)=T(\bar{G})=T(\bar{F})$. Таким образом, при всех $s=0,1, \ldots, 2^{\nu}-1$ последовательность $v_{s}$ является ненулевой ЛРП с реверсивным характеристическим многочленом Галуа $G(x)$, для которого $T(G)=T(\bar{G})=t$. Тогда, используя лемму 5, получим

$$
\left|\sum_{i=0}^{l-1} \chi(\nu(i))\right| \leq \sum_{s=0}^{2^{\nu}-1}\left|\sum_{i=0}^{\left[\frac{l-1-s}{2^{\nu}}\right]} \chi\left(v_{s}(i)\right)\right| \leq\left(\frac{2}{\pi} \ln \left(\frac{4 t}{\pi}\right)+1\right) 2^{\frac{m}{2}+\nu+n-1} .
$$

Докажем основной результат этого параграфа.

Теорема 4. Если $F(x)$ - реверсивный многочлен Галуа степени $m$ над кольцом $R=\mathbb{Z}_{2^{n}}, n \geq 2, T(F)=2^{\nu} T(\bar{F})=2^{\nu} t, u \in L_{R}(F)^{*}, K-$ координатное множество кольия $\mathbb{Z}_{2^{n}}, u_{n-1}=\varkappa_{n-1}^{K}(u), l \leq T(F), z \in K$, то справедлива оченка

$$
\left|N_{l}\left(z, u_{n-1}\right)-\frac{l}{2}\right|<\left(\frac{1}{\pi} \ln \left(2^{n-1}\right)+\frac{1}{2}\right)\left(\frac{2}{\pi} \ln \left(\frac{4 t}{\pi}\right)+1\right) 2^{\frac{m}{2}+\nu+n-1} .
$$

Доказательство. В силу утверждения 3

$$
N_{l}\left(z, u_{n-1}\right)-\frac{l}{2}=\frac{(-1)^{z}}{2} \sum_{i=0}^{l-1}(-1)^{\varkappa_{n-1}^{K}(u(i))} .
$$

Воспользовавшись равенством (3) и изменяя порядок суммирования, получим

$$
N_{l}\left(z, u_{n-1}\right)-\frac{l}{2}=\frac{(-1)^{z}}{2} \sum_{i=0}^{l-1} \sum_{j=0}^{2^{n}-1} \mu_{j} \chi(u(i) j)=\frac{(-1)^{z}}{2} \sum_{j=0}^{2^{n}-1} \mu_{j} \sum_{i=0}^{l-1} \chi(u(i) j) .
$$

Так как $\mu_{0}=0$, то

$$
\left|N_{l}\left(z, u_{n-1}\right)-\frac{l}{2}\right| \leq \frac{1}{2}\left(\sum_{j=0}^{2^{n}-1}\left|\mu_{j}\right|\right) \max _{v \in L_{R}(F), v \neq(0)}\left|\sum_{i=0}^{l-1} \chi(v(i))\right| .
$$


Для завершения доказательства теоремы остается воспользоваться теоремой 1 и леммой 6.

Раньше в [12, теоремы 5 и 6] для частного случая, когда $K=\{0,1\}$, $n \geq 4, \bar{F}(x)$ - многочлен максимального периода над полем $G F(2)$, а $\nu$ принимает значения 0 или $n-1$, была получена оценка, аналогичная оценке из теоремы 4 (она отличается лишь заменой множителя $(1 / \pi) \ln \left(2^{n-1}\right)+1 / 2$ величиной $\left.(1 / \pi) \ln \left(2^{n}\right)+1 / 2\right)$. Таким образом, теорема 4 не только обобщает указанные результаты, но и уточняет их.

\section{6. Асимптотическая неулучшаемость оценок}

В данном параграфе рассмотрим вопрос о том, насколько точными являются оценки, полученные в предыдущих параграфах.

Лемма 7. Для чисел $\mu_{j}$, определенных равенством (4), справедливы соотношения

$$
\sum_{j=0}^{2^{n}-1}\left|\mu_{j}\right|^{2}=1, \quad \sum_{s=0}^{2^{n}-1} \bar{\mu}_{s} \mu_{s+2^{n-1}}=0
$$

где $\bar{\mu}_{s}-$ комплексное число, сопряженное с числом $\mu_{s}, s=0,1, \ldots, 2^{n}-1$.

Доказательство. Из определения спектральных коэффициентов $\mu_{j}$ следуют равенства

$$
\begin{aligned}
\sum_{j=0}^{2^{n}-1}\left|\mu_{j}\right|^{2} & =\sum_{j=0}^{2^{n}-1} \mu_{j} \bar{\mu}_{j}= \\
& =\sum_{j=0}^{2^{n}-1}\left(\frac{1}{2^{n}} \sum_{s=0}^{2^{n}-1}(-1)^{\varkappa_{n-1}^{K}(s)} \chi(-s j)\right)\left(\frac{1}{2^{n}} \sum_{k=0}^{2^{n}-1}(-1)^{\varkappa_{n-1}^{K}(k)} \chi(k j)\right) .
\end{aligned}
$$

Изменив порядок суммирования, получим

$$
\sum_{j=0}^{2^{n}-1}\left|\mu_{j}\right|^{2}=\frac{1}{2^{2 n}} \sum_{s=0}^{2^{n}-1}(-1)^{\varkappa_{n-1}^{K}(s)} \sum_{k=0}^{2^{n}-1}(-1)^{\varkappa_{n-1}^{K}(k)} \sum_{j=0}^{2^{n}-1} \chi((k-s) j) ;
$$

отсюда и из (5) следует, что

$$
\sum_{j=0}^{2^{n}-1}\left|\mu_{j}\right|^{2}=\frac{1}{2^{n}} \sum_{s=0}^{2^{n}-1}(-1)^{2 \varkappa_{n-1}^{K}(s)}=1 .
$$


Докажем второе соотношение. Справедливо равенство

$$
\begin{aligned}
& \sum_{s=0}^{2^{n}-1} \bar{\mu}_{s} \mu_{s+2^{n-1}}= \\
& =\sum_{s=0}^{2^{n}-1}\left(\frac{1}{2^{n}} \sum_{i=0}^{2^{n}-1}(-1)^{\varkappa_{n-1}^{K}(i)} \chi(i s)\right)\left(\frac{1}{2^{n}} \sum_{k=0}^{2^{n}-1}(-1)^{\varkappa_{n-1}^{K}(k)} \chi\left(-k\left(s+2^{n-1}\right)\right)\right) .
\end{aligned}
$$

Изменив порядок суммирования, получим

$$
\begin{aligned}
& \sum_{s=0}^{2^{n}-1} \bar{\mu}_{s} \mu_{s+2^{n-1}}= \\
& \quad=\frac{1}{2^{2 n}} \sum_{k=0}^{2^{n}-1}(-1)^{\varkappa_{n-1}^{K}(k)} \chi\left(-k 2^{n-1}\right) \sum_{i=0}^{2^{n}-1}(-1)^{\varkappa_{n-1}^{K}(i)} \sum_{s=0}^{2^{n}-1} \chi((i-k) s) ;
\end{aligned}
$$

отсюда и из соотношения ортогональности (5) следует, что

$$
\sum_{s=0}^{2^{n}-1} \bar{\mu}_{s} \mu_{s+2^{n-1}}=\frac{1}{2^{n}} \sum_{k=0}^{2^{n}-1}(-1)^{2 \varkappa_{n-1}^{K}(k)} \chi\left(-k 2^{n-1}\right)=\frac{1}{2^{n}} \sum_{k=0}^{2^{n}-1}(-1)^{-k}=0 .
$$

Теорема 5. Если $F(x)$ - реверсивный многочлен Галуа степени $m$ над кольиом $R=\mathbb{Z}_{2^{n}}, n \geq 2, K$ - координатное множество кольиа $\mathbb{Z}_{2^{n}}$, то для любых $l \leq T(\bar{F}), z \in K$ найдется такая ЛРП $u \in L_{R}(F)^{*}$, что для последовательности $u_{n-1}=\varkappa_{n-1}^{K}(u)$ справедливо неравенство

$$
\left|N_{l}\left(z, u_{n-1}\right)-\frac{l}{2}\right| \geq \frac{\sqrt{l}}{2} .
$$

Доказательство. При доказательстве теоремы 4 было показано, что

$$
\left|N_{l}\left(z, u_{n-1}\right)-\frac{l}{2}\right|=\frac{1}{2}\left|S_{l}(u)\right|,
$$

где

$$
S_{l}(u)=\sum_{j=0}^{2^{n}-1} \mu_{j} \sum_{i=0}^{l-1} \chi(u(i) j)
$$

2010, T. 1, № 4, C. 33-62 
Положим $\Delta=\sum_{u \in L_{R}(F)^{*}}\left|S_{l}(u)\right|^{2}$, тогда найдется ЛРП $u \in L_{R}(F)^{*}$ такая, что для последовательности $u_{n-1}=\varkappa_{n-1}^{K}(u)$ справедливо соотношение

$$
\left|N_{l}\left(z, u_{n-1}\right)-\frac{l}{2}\right| \geq \frac{1}{2}\left(\frac{\Delta}{\left|L_{R}(F)^{*}\right|}\right)^{\frac{1}{2}}=\frac{1}{2}\left(\frac{\Delta}{2^{m(n-1)}\left(2^{m}-1\right)}\right)^{\frac{1}{2}} .
$$

Справедливы равенства

$$
\begin{aligned}
\Delta=\sum_{u \in L_{R}(F)^{*}} S_{l}(u) \bar{S}_{l}(u)= & \\
& =\sum_{u \in L_{R}(F)^{*}}\left(\sum_{j=0}^{2^{n}-1} \mu_{j} \sum_{i=0}^{l-1} \chi(u(i) j)\right)\left(\sum_{s=0}^{2^{n}-1} \bar{\mu}_{s} \sum_{k=0}^{l-1} \chi(-u(k) s)\right) .
\end{aligned}
$$

Изменив порядок суммирования, получим

$$
\Delta=\sum_{j, s=0}^{2^{n}-1} \mu_{j} \bar{\mu}_{s} \sum_{i, k=0}^{l-1} \sum_{u \in L_{R}(F)^{*}} \chi(u(i) j-u(k) s) .
$$

Обозначим через $S=G R\left(2^{m n}, 2^{n}\right)$ кольцо Галуа, являющееся расширением степени $m$ кольца $R$. Каждая последовательность $u$ из множества $L_{R}(F)^{*}$ однозначно представима в виде

$$
u(i)=\operatorname{Tr}_{R}^{S}\left(b \alpha^{i}\right),
$$

где $\operatorname{Tr}_{R}^{S}$ - функция след из кольца $S$ в кольцо $R, \alpha-$ корень многочлена $F(x)$ в кольце $S, b-$ обратимый элемент кольца $S$. Если $\chi^{\prime}-$ аддитивный характер кольца $S$, определенный на элементах этого кольца равенством $\chi^{\prime}(y)=\chi\left(\operatorname{Tr}_{R}^{S}(y)\right)$, то

$$
\sum_{u \in L_{R}(F)^{*}} \chi(u(i) j-u(k) s)=\sum_{b \in S^{*}} \chi^{\prime}\left(b\left(\alpha^{i} j-\alpha^{k} s\right)\right),
$$

и согласно равенству (5) из работы [6]

$$
\sum_{u \in L_{R}(F)^{*}} \chi(u(i) j-u(k) s)= \begin{cases}\left|S^{*}\right|, & \text { если } \alpha^{i} j-\alpha^{k} S=0, \\ -|2 S|, & \text { если } \alpha^{i} j-\alpha^{k} S \in 2^{n-1} S \backslash\{0\}, \\ 0, & \text { если } \alpha^{i} j-\alpha^{k} S \notin 2^{n-1} S .\end{cases}
$$

Так как числа $\mu_{t}$ равны 0 при всех четных значениях $t$, то в равенстве (9) достаточно рассматривать лишь случай, когда $j$ и $s-$ обратимые элементы 
кольца $R$. При таких $j$ и $s$ каждое из соотношений $\alpha^{i} j-\alpha^{k} s=0$ и $\alpha^{i} j-\alpha^{k} s \in$ $\in 2^{n-1} S \backslash\{0\}$ влечет равенство $\bar{\alpha}^{k-i}=1$, где $\bar{\alpha}-$ образ элемента $\alpha$ при действии естественного эпиморфизма из кольца $S$ в поле $S /(2 S)$. Это возможно только в случае, когда $T(\bar{F})$ делит $k-i$, что при $l \leq T(\bar{F})$ равносильно условию $k=i$. Из неравенства (9) следует, что

$$
\begin{aligned}
& \Delta=\sum_{j=0}^{2^{n}-1} \mu_{j} \bar{\mu}_{j} \sum_{i=0}^{l-1}\left|S^{*}\right|+\sum_{s=0}^{2^{n}-1} \mu_{s+2^{n-1}} \bar{\mu}_{s} \sum_{i=0}^{l-1}(-|2 S|)= \\
&=\left|S^{*}\right| l \sum_{j=0}^{2^{n}-1}\left|\mu_{j}\right|^{2}-|2 S| l \sum_{s=0}^{2^{n}-1} \bar{\mu}_{s} \mu_{s+2^{n-1}}
\end{aligned}
$$

С учетом леммы 7 получим $\Delta=2^{m(n-1)}\left(2^{m}-1\right) l$, а тогда в силу (8)

$$
\left|N_{l}\left(z, u_{n-1}\right)-\frac{l}{2}\right| \geq \frac{\sqrt{l}}{2} \text {. }
$$

Теорема 5 показывает, что для любых $z \in K, l \leq T(\bar{F})$ и произвольного координатного множества $K$ в классе $L_{R}(F)^{*}$ найдется ЛРП $u$, у которой в старшей координатной последовательности $u_{n-1}$ частота $N_{l}\left(z, u_{n-1}\right)$ отклоняется от среднего значения на величину не меньше $\sqrt{l} / 2$. Вопрос о том, может ли это отклонение быть значительно большим, остается открытым. Результаты следствия 1 о распределении элементов на циклах старших координатных последовательностей отмеченных ЛРП над кольцом $\mathbb{Z}_{4}$ показывают, что для всех таких последовательностей отклонение величины $N_{l}\left(z, u_{n-1}\right)$ от $l / 2$ при $l=T(\bar{F})$ практически совпадает с числом $\sqrt{l} / 2$ либо меньше этого числа.

Рассмотрим реверсивный многочлен Галуа $F(x)$ степени $m$ над кольцом $\mathbb{Z}_{2^{n}}$, для которого $T(\bar{F})=2^{m}-1$. Как показано в теореме 5 , при $l=$ $=T(\bar{F})$ для каждого элемента $z \in K$ найдется такая ЛРП $u \in L_{R}(F)^{*}$, что для последовательности $u_{n-1}=\varkappa_{n-1}^{K}(u)$ справедливо неравенство

$$
\left|N_{l}\left(z, u_{n-1}\right)-\frac{l}{2}\right| \geq \frac{\sqrt{2^{m}-1}}{2} .
$$

Это означает, что в оценках из теорем 2 и 4 главный множитель $2^{m / 2}$ нельзя заменить множителем $a^{m / 2}$, где $a<2$. Таким образом, эти оценки являются асимптотически неулучшаемыми в классе всех старших координатных последовательностей ЛРП из множества $L_{R}(F)^{*}$. 


\section{7. Автокорреляционная функция старшей координатной последовательности}

Пусть $u$ - последовательность над кольцом $R=\mathbb{Z}_{2^{n}}, K$ - координатное множество кольца $K, u_{n-1}=\varkappa_{n-1}^{K}(u)-$ старшая координатная последовательность последовательности $u$. Автокорреляционная функция последовательности $u_{n-1}$ для всех целых неотрицательных чисел $l$ и $\tau$ определяется равенством

$$
C(l, \tau)=\sum_{i=0}^{l-1}(-1)^{u_{n-1}(i)-u_{n-1}(i+\tau)} .
$$

Очевидно, что величина $C(l, \tau)$ равна разности числа совпадающих координат в векторах $\left(u_{n-1}(0), u_{n-1}(1), \ldots, u_{n-1}(l-1)\right),\left(u_{n-1}(\tau), u_{n-1}(\tau+\right.$ $\left.+1), \ldots, u_{n-1}(\tau+l-1)\right)$ и числа несовпадающих координат в этих векторах.

Теорема 6. Если $F(x)$ - реверсивный многочлен Галуа степени $m$ над кольиом $R=\mathbb{Z}_{2^{n}}, T(F)=2^{\nu} T(\bar{F})=2^{\nu}\left(2^{m}-1\right) / d, T(\bar{F})=t, u \in L_{R}(F)$, $u \neq(0), K-$ координатное множество кольиа $\mathbb{Z}_{2^{n}}, u_{n-1}=\varkappa_{n-1}^{K}(u)$, то для всех $\tau \in \mathbb{N}_{0}$, которые не делятся на $t$ :

1) если $l=T(F)$, то

$$
|C(l, \tau)| \leq\left(\frac{2}{\pi} \ln \left(2^{n-1}\right)+1\right)^{2} \frac{2^{\nu}}{d}\left(\left(d 2^{n-1}-1\right) 2^{\frac{m}{2}}+1\right) ;
$$

2) если $l<T(F)$, то

$$
|C(l, \tau)| \leq\left(\frac{2}{\pi} \ln \left(2^{n-1}\right)+1\right)^{2}\left(\frac{2}{\pi} \ln \left(\frac{4 t}{\pi}\right)+1\right) 2^{\frac{m}{2}+n+\nu-1} .
$$

Доказательство. Из равенства

$$
C(l, \tau)=\sum_{i=0}^{l-1}(-1)^{u_{n-1}(i)-u_{n-1}(i+\tau)}=\sum_{i=0}^{l-1}(-1)^{\varkappa_{n-1}^{K}(u(i))}(-1)^{\varkappa_{n-1}^{K}(u(i+\tau))}
$$

с помощью соотношения (3) получим

$$
\begin{aligned}
C(l, \tau)=\sum_{i=0}^{l-1} \sum_{j_{1}=0}^{2^{n}-1} \mu_{j_{1}} \chi\left(u(i) j_{1}\right) \sum_{j_{2}=0}^{2^{n}-1} \mu_{j_{2}} \chi\left(u(i+\tau) j_{2}\right)= \\
=\sum_{j_{1}, j_{2}=0}^{2^{n}-1} \mu_{j_{1}} \mu_{j_{2}} \sum_{i=0}^{l-1} \chi\left(v_{j_{1}, j_{2}, \tau}(i)\right),
\end{aligned}
$$


где $v_{j_{1}, j_{2}, \tau}-$ последовательность элементов кольца $\mathbb{Z}_{2^{n}}$, определенная при всех $\dot{j}_{1}, \dot{j}_{2} \in \mathbb{Z}_{2^{n}}, \tau \in \mathbb{N}_{0}$ равенством

$$
v_{j_{1}, j_{2}, \tau}(i)=u(i) j_{1}+u(i+\tau) j_{2}, i \geq 0 .
$$

Пусть представление ЛРП $u$ в терминах функции след имеет вид

$$
u(i)=\operatorname{Tr}_{R}^{S}\left(b \alpha^{i}\right)
$$

где $S=G R\left(2^{m n}, 2^{n}\right)$ - кольцо Галуа, являющееся расширением степени $m$ кольца $R, \operatorname{Tr}_{R}^{S}$ - функция след из кольца $S$ в кольцо $R, \alpha$ - корень многочлена $F(x)$ в кольце $S, b-$ ненулевой элемент кольца $S$. Последовательность $v_{j_{1}, j_{2}, \tau}$ представима в виде

$$
v_{j_{1}, j_{2}, \tau}=\operatorname{Tr}_{R}^{S}\left(b\left(j_{1}+\alpha^{\tau} j_{2}\right) \alpha^{i}\right), i \geq 0
$$

Согласно лемме 1 коэффициенты $\mu_{j}=0$ при всех $j \in 2 \mathbb{Z}_{2^{n}}$, поэтому в соотношениях (10) достаточно рассматривать суммирование по всем $j_{1}, j_{2} \in \mathbb{Z}_{2^{n}}$, обратимым в этом кольце. Обозначим через $\bar{\alpha}$ образ элемента $\alpha$ при действии естественного эпиморфизма из кольца $S$ в фактор-кольцо $S /(2 S)=G F\left(2^{m}\right)$. Если бы элемент $j_{1}+\alpha^{\tau} j_{2}$ принадлежал множеству $2 S$, то имело бы место равенство $\bar{\alpha}^{\tau}=1$, что невозможно при $\tau$, не делящихся на $t=T(\bar{F})$. Таким образом, при всех $j_{1}, j_{2} \in \mathbb{Z}_{2^{n}}^{*}$ элемент $b\left(j_{1}+\alpha^{\tau} j_{2}\right)$ отличен от нуля, а значит, $v_{j_{1}, j_{2}, \tau}-$ ненулевая последовательность из множества $L_{R}(F)$ всех ЛРП над кольцом $R$ с характеристическим многочленом $F(x)$. Тогда из (10) получим

$$
|C(l, \tau)| \leq \sum_{j_{1}, j_{2}=0}^{2^{n}-1}\left|\mu_{j_{1}} \mu_{j_{2}}\right| \max _{j_{1}, j_{2} \in R^{*}}\left|\sum_{i=0}^{l-1} \chi\left(v_{j_{1}, j_{2}, \tau}(i)\right)\right|,
$$

а в силу теоремы 1

$$
|C(l, \tau)| \leq\left(\frac{2}{\pi} \ln \left(2^{n-1}\right)+1\right)^{2} \max _{v \in L_{R}(F), v \neq(0)}\left|\sum_{i=0}^{l-1} \chi(v(i))\right| .
$$

Теперь для доказательства п. 1) теоремы достаточно воспользоваться леммой 2, а для доказательства п. 2) - леммой 5 .

Теорема 6 уточняет и обобщает оценки коэффициентов автокорреляции, полученные в работах [8, теорема 3.5] и [12, теоремы 9 и 10]. 


\section{8. Обобщения на случай $r$-грамм}

В этом параграфе обобщим результаты о распределении элементов в старшей координатной последовательности на случай координатных последовательностей ЛРП векторов, в частности, отсюда будут следовать результаты о распределении $r$-грамм в старших координатных последовательностях.

Рассмотрим ЛРП $u_{1}, u_{2}, \ldots, u_{r}$ над кольцом $R=\mathbb{Z}_{2^{n}}$, имеющие один и тот же характеристический многочлен $F(x)$. ЛРП векторов будем называть последовательность, элементы которой имеют вид $\left(u_{1}(i), u_{2}(i), \ldots, u_{r}(i)\right)$ для всех $i \geq 0$. Систему последовательностей $u_{1}, u_{2}, \ldots, u_{r}$ будем называть линейно независимой над кольцом $R$, если для всех ненулевых наборов $\left(c_{1}, c_{2}, \ldots, c_{r}\right) \in R^{r}$ последовательность $c_{1} u_{1}+c_{2} u_{2}+\ldots+c_{r} u_{r}$ является ненулевой.

Пусть $K-$ координатное множество кольца $R, u_{n-1}^{(s)}=\varkappa_{n-1}^{K}\left(u_{s}\right)-$ старшая координатная последовательность последовательности $u_{s}$, где $s=$ $=1,2, \ldots, r$. Последовательность из наборов $\left(u_{n-1}^{(1)}(i), u_{n-1}^{(2)}(i), \ldots, u_{n-1}^{(r)}(i)\right)$, где $i \geq 0$, будем называть старшей координатной последовательностью ЛРП векторов.

Для каждого натурального числа $l$ и набора $\bar{z}=\left(z_{1}, z_{2}, \ldots, z_{r}\right)$ из множества $K^{r}$ обозначим через $N_{l}\left(\bar{z}, u_{n-1}^{(1)}, \ldots, u_{n-1}^{(r)}\right)$ количество таких целых чисел $i \in \overline{0, l-1}$, что

$$
\left\{\begin{array}{c}
u_{n-1}^{(1)}(i)=z_{1}, \\
u_{n-1}^{(2)}(i)=z_{2}, \\
\cdots \\
u_{n-1}^{(r)}(i)=z_{r} .
\end{array}\right.
$$

Другими словами, величина $N_{l}\left(\bar{z}, u_{n-1}^{(1)}, \ldots, u_{n-1}^{(r)}\right)$ равна числу появлений $r$-граммы $\bar{z}$ на начальном отрезке длины $l$ старшей координатной последовательности рассматриваемой ЛРП векторов. Отметим, что если последовательности $u_{1}, u_{2}, \ldots, u_{r}$ являются сдвигами одной ЛРП $u$, т. е. для некоторых целых неотрицательных чисел $s_{1}, s_{2}, \ldots, s_{r}$ выполнены равенства $u_{1}=x^{s_{1}} u$, $u_{2}=x^{s_{2}} u, \ldots, u_{r}=x^{s_{r}} u$, то величина $N_{l}\left(\bar{z}, u_{n-1}^{(1)}, \ldots, u_{n-1}^{(r)}\right)$ равна числу появлений вектора $\bar{z}$ среди векторов $\left(u_{n-1}\left(i+s_{1}\right), u_{n-1}\left(i+s_{2}\right), \ldots, u_{n-1}\left(i+s_{r}\right)\right)$, где $0 \leq i \leq l-1$. Таким образом, из результатов о распределении наборов $\bar{z}$ в старшей координатной последовательности ЛРП векторов следуют результаты о распределении $r$-грамм в старшей координатной последовательности одной ЛРП. 
Теорема 7. Если $F(x)$ - реверсивный многочлен Галуа степени $m$ над кольиом $R=\mathbb{Z}_{2^{n}}, T(F)=2^{\nu}\left(2^{m}-1\right) / d, 0 \leq \nu \leq n-1, u_{1}, u_{2}, \ldots, u_{r} \in$ $\in L_{R}(F)$ - линейно независимая система, $K$ - координатное множество кольиа $\mathbb{Z}_{2^{n}}, u_{n-1}^{(s)}=\varkappa_{n-1}^{K}\left(u_{s}\right)$, где $s=1,2, \ldots, r, \bar{z} \in K^{r}$, то для числа $N\left(\bar{z}, u_{n-1}^{(1)}, \ldots, u_{n-1}^{(r)}\right)=N_{T(F)}\left(\bar{z}, u_{n-1}^{(1)}, \ldots, u_{n-1}^{(r)}\right)$ справедлива оченка

$$
\left|N\left(\bar{z}, u_{n-1}^{(1)}, \ldots, u_{n-1}^{(r)}\right)-\omega(\bar{z})\right| \leq\left(\left(\frac{1}{\pi} \ln \left(2^{n-1}\right)+1\right)^{r}-\frac{1}{2^{r}}\right) \frac{2^{\nu}\left(d 2^{n-1}-1\right)}{d} 2^{\frac{m}{2}},
$$

где

$$
\omega(\bar{z})= \begin{cases}\frac{2^{m-r}-1}{2^{m}-1} T(F), & \text { если } \bar{z}=\left(\varkappa_{n-1}^{K}(0), \ldots, \varkappa_{n-1}^{K}(0)\right), \\ \frac{2^{m-r}}{2^{m}-1} T(F), & \text { если } \bar{z} \neq\left(\varkappa_{n-1}^{K}(0), \ldots, \varkappa_{n-1}^{K}(0)\right) .\end{cases}
$$

Доказательство. Из равенств (6) следует, что

$$
N\left(\bar{z}, u_{n-1}^{(1)}, \ldots, u_{n-1}^{(r)}\right)=\sum_{i=0}^{T(F)-1} \prod_{j=1}^{r}\left(\frac{1}{2} \sum_{c_{j}=0}^{1}(-1)^{c_{j}\left(u_{n-1}^{(j)}(i)+z_{j}\right)}\right)
$$

Поэтому

$$
\begin{aligned}
& N\left(\bar{z}, u_{n-1}^{(1)}, \ldots, u_{n-1}^{(r)}\right)= \\
& \quad=\frac{1}{2^{r}} \sum_{i=0}^{T(F)-1} \sum_{\left(c_{1}, \ldots, c_{r}\right) \in\{0,1\}^{r}}(-1)^{c_{1} u_{n-1}^{(1)}(i)+\ldots+c_{r} u_{n-1}^{(r)}(i)}(-1)^{c_{1} z_{1}+\ldots+c_{r} z_{r}} .
\end{aligned}
$$

Изменив порядок суммирования и выделив отдельно слагаемое, соответствующее нулевому набору $\bar{c}=\left(c_{1}, \ldots, c_{r}\right)=(0, \ldots, 0)=\overline{0}$, получим:

$$
\begin{aligned}
N\left(\bar{z}, u_{n-1}^{(1)}, \ldots, u_{n-1}^{(r)}\right)-\frac{T(F)}{2^{r}}= \\
=\frac{1}{2^{r}} \sum_{\left(c_{1}, \ldots, c_{r}\right) \neq \overline{0}}(-1)^{c_{1} z_{1}+\ldots+c_{r} z_{r}} \sum_{i=0}^{T(F)-1}(-1)^{c_{1} u_{n-1}^{(1)}(i)+\ldots+c_{r} u_{n-1}^{(r)}(i)} .
\end{aligned}
$$


Разобьем правую часть на суммы по всем векторам $\bar{c} \in\{0,1\}^{r}$ веса $k$, где $k=1,2, \ldots, r$ :

$$
\begin{aligned}
& N\left(\bar{z}, u_{n-1}^{(1)}, \ldots, u_{n-1}^{(r)}\right)-\frac{T(F)}{2^{r}}= \\
& =\frac{1}{2^{r}} \sum_{k=1}^{r} \sum_{\left\{t_{1}, \ldots, t_{k}\right\} \subset \overline{1, r}}(-1)^{\sum_{s=1}^{k} z_{t_{s}}} \sum_{i=0}^{T(F)-1}(-1)^{\sum_{s=1}^{k} u_{n-1}^{\left(t_{s}\right)}(i)} .
\end{aligned}
$$

В силу (3)

$$
\sum_{i=0}^{T(F)-1}(-1)^{\sum_{s=1}^{k} u_{n-1}^{\left(t_{s}\right)}(i)}=\sum_{i=0}^{T(F)-1}\left(\sum_{j_{1}=0}^{2^{n}-1} \mu_{j_{1}} \chi\left(u_{t_{1}}(i) j_{1}\right)\right) \cdots\left(\sum_{j_{k}=0}^{2^{n}-1} \mu_{j_{k}} \chi\left(u_{t_{k}}(i) j_{k}\right)\right)
$$

а значит,

$$
\sum_{i=0}^{T(F)-1}(-1)^{\sum_{s=1}^{k} u_{n-1}^{\left(t_{s}\right)}(i)}=\sum_{\left(j_{1}, \ldots, j_{k}\right) \in R^{k}} \mu_{j_{1}} \ldots \mu_{j_{k}} \sum_{i=0}^{T(F)-1} \chi\left(v_{t_{1}, \ldots, t_{k}, j_{1}, \ldots, j_{k}}(i)\right),
$$

где $v_{t_{1}, \ldots, t_{k}, j_{1}, \ldots, j_{k}}-$ последовательность над кольцом $R$, элементы которой определены равенством

$$
v_{t_{1}, \ldots, t_{k}, j_{1}, \ldots, j_{k}}(i)=u_{t_{1}}(i) j_{1}+\ldots+u_{t_{k}}(i) j_{k}, \quad i \geq 0 .
$$

Тогда из равенства (11) следует, что

$$
\begin{gathered}
N\left(\bar{z}, u_{n-1}^{(1)}, \ldots, u_{n-1}^{(r)}\right)-\delta(\bar{z})= \\
=\frac{1}{2^{r}} \sum_{k=1}^{r} \sum_{\left\{t_{1}, \ldots, t_{k}\right\} \subset \overline{1, r}}(-1)^{\sum_{s=1}^{k} z_{t_{s}}} \sum_{\left(j_{1}, \ldots, j_{k}\right) \in R^{k}} \mu_{j_{1}} \ldots \mu_{j_{k}}\left(\sum_{i=0}^{T(F)-1} \chi\left(v_{t_{1}, \ldots, t_{k}, j_{1}, \ldots, j_{k}}(i)\right)+\frac{2^{\nu}}{d}\right),
\end{gathered}
$$

где

$$
\delta(\bar{z})=\frac{T(F)}{2^{r}}-\frac{2^{\nu}}{d 2^{r}} \sum_{k=1}^{r} \sum_{\left\{t_{1}, \ldots, t_{k}\right\} \subset \overline{1, r}}(-1)^{\sum_{s=1}^{k} z_{t_{s}}} \sum_{\left(j_{1}, \ldots, j_{k}\right) \in R^{k}} \mu_{j_{1}} \ldots \mu_{j_{k}} .
$$

Заметим, что в силу линейной независимости системы $u_{1}, u_{2}, \ldots, u_{r}$ последовательность $v_{t_{1}, \ldots, t_{k}, j_{1}, \ldots, j_{k}}$ является ненулевой ЛРП с характеристическим многочленом $F(x)$ при всех ненулевых наборах $\left(j_{1}, \ldots, j_{k}\right) \in R^{k}$ и при всех 
$\left\{t_{1}, \ldots, t_{k}\right\} \subset \overline{1, r}$. Кроме того, согласно лемме 1 коэффициент $\mu_{0}=0$, поэтому справедливо неравенство

$$
\begin{aligned}
& \left|N\left(\bar{z}, u_{n-1}^{(1)}, \ldots, u_{n-1}^{(r)}\right)-\delta(\bar{z})\right| \leq \\
\leq & \frac{1}{2^{r}} \sum_{k=1}^{r} \sum_{\left\{t_{1}, \ldots, t_{k}\right\} \subset \overline{1, r}}\left(\sum_{\left(j_{1}, \ldots, j_{k}\right) \in R^{k}}\left|\mu_{j_{1}} \ldots \mu_{j_{k}}\right|\right) \max _{v \in L_{R}(F), v \neq(0)}\left|\sum_{i=0}^{T(F)-1} \chi(v(i))+\frac{2^{\nu}}{d}\right| .
\end{aligned}
$$

Используя теорему 1 и лемму 2, получим

$$
\begin{aligned}
\mid N\left(\bar{z}, u_{n-1}^{(1)}, \ldots, u_{n-1}^{(r)}\right) & -\delta(\bar{z}) \mid \leq \\
& \leq \frac{1}{2^{r}} \sum_{k=1}^{r}\left(\begin{array}{l}
r \\
k
\end{array}\right)\left(\frac{2}{\pi} \ln \left(2^{n-1}\right)+1\right)^{k} \frac{2^{\nu}\left(d 2^{n-1}-1\right)}{d} 2^{\frac{m}{2}},
\end{aligned}
$$

а значит, учитывая формулу бинома Ньютона, имеет место неравенство

$$
\left|N\left(\bar{z}, u_{n-1}^{(1)}, \ldots, u_{n-1}^{(r)}\right)-\delta(\bar{z})\right| \leq\left(\left(\frac{1}{\pi} \ln \left(2^{n-1}\right)+1\right)^{r}-\frac{1}{2^{r}}\right) \frac{2^{\nu}\left(d 2^{n-1}-1\right)}{d} 2^{\frac{m}{2}} .
$$

Вычислим величину $\delta(\bar{z})$, определенную равенством (12). Представим ее следующим образом:

$$
\delta(\bar{z})=\frac{T(F)}{2^{r}}-\frac{2^{\nu}}{d 2^{r}} \sum_{k=1}^{r} \sum_{\left\{t_{1}, \ldots, t_{k}\right\} \subset \overline{1, r}}(-1)^{\sum_{s=1}^{k} z_{t_{s}}}\left(\sum_{j=0}^{2^{n}-1} \mu_{j}\right)^{k} .
$$

Отсюда и из леммы 3 следует, что

$$
\delta(\bar{z})=\frac{T(F)}{2^{r}}-\frac{2^{\nu}}{d 2^{r}} \sum_{k=1}^{r}(-1)^{k x_{n-1}^{K}(0)} \sum_{\left\{t_{1}, \ldots, t_{k}\right\} \subset \overline{1, r}}(-1)^{\sum_{s=1}^{k} z_{t_{s}}},
$$

а значит,

$$
\delta(\bar{z})=\frac{T(F)}{2^{r}}-\frac{2^{\nu}}{d 2^{r}} \sum_{\left(c_{1}, \ldots, c_{r}\right) \neq \overline{0}}(-1)^{c_{1}\left(\varkappa_{n-1}^{K}(0)+z_{1}\right)} \ldots(-1)^{c_{r}\left(\varkappa_{n-1}^{K}(0)+z_{r}\right)} .
$$

Тогда

$\delta(\bar{z})=\frac{T(F)}{2^{r}}-\frac{2^{\nu}}{d 2^{r}}\left(\sum_{c_{1}=0}^{1}(-1)^{c_{1}\left(\varkappa_{n-1}^{K}(0)+z_{1}\right)}\right) \ldots\left(\sum_{c_{r}=0}^{1}(-1)^{c_{r}\left(\varkappa_{n-1}^{K}(0)+z_{r}\right)}\right)+\frac{2^{\nu}}{d 2^{r}}$, 
и в силу равенств (6)

$$
\delta(\bar{z})= \begin{cases}\frac{T(F)}{2^{r}}-\frac{2^{\nu}\left(2^{r}-1\right)}{d 2^{r}}, & \text { если } \bar{z}=\left(\varkappa_{n-1}^{K}(0), \ldots, \varkappa_{n-1}^{K}(0)\right), \\ \frac{T(F)}{2^{r}}+\frac{2^{\nu}}{d 2^{r}}, & \text { если } \bar{z} \neq\left(\varkappa_{n-1}^{K}(0), \ldots, \varkappa_{n-1}^{K}(0)\right) .\end{cases}
$$

Учитывая, что $T(F)=2^{\nu}\left(2^{m}-1\right) / d$, получим $\delta(\bar{z})=\omega(\bar{z})$.

Рассмотрим вопрос о появлении векторов $\bar{z}$ на произвольных отрезках старших координатных последовательностей ЛРП векторов.

Теорема 8. Если $F(x)$ - реверсивный многочлен Галуа степени $m$ над кольиом $R=\mathbb{Z}_{2^{n}}, T(F)=2^{\nu} T(\bar{F}), 0 \leq \nu \leq n-1, t=T(\bar{F})$, $u_{1}, u_{2}, \ldots, u_{r} \in L_{R}(F)$ - линейно независимая система, $K-$ координатное множество кольияа $\mathbb{Z}_{2^{n}, u_{n-1}^{(s)}}=\varkappa_{n-1}^{K}\left(u_{s}\right)$, где $s=1,2, \ldots, r, \bar{z} \in K^{r}, l \leq T(F)$, то справедлива оценка

$$
\begin{aligned}
\left|N_{l}\left(\bar{z}, u_{n-1}^{(1)}, \ldots, u_{n-1}^{(r)}\right)-\frac{l}{2^{r}}\right| \leq \\
\quad \leq\left(\left(\frac{1}{\pi} \ln \left(2^{n-1}\right)+1\right)^{r}-\frac{1}{2^{r}}\right)\left(\frac{2}{\pi} \ln \left(\frac{4 t}{\pi}\right)+1\right) 2^{\frac{m}{2}+n+\nu-1} .
\end{aligned}
$$

Доказательство. С помощью равенств (6) получим равенство

$$
N_{l}\left(\bar{z}, u_{n-1}^{(1)}, \ldots, u_{n-1}^{(r)}\right)=\sum_{i=0}^{l-1} \prod_{j=1}^{r}\left(\frac{1}{2} \sum_{c_{j}=0}^{1}(-1)^{c_{j}\left(u_{n-1}^{(j)}(i)+z_{j}\right)}\right) .
$$

Тогда, по аналогии с доказательством теоремы 7, имеет место соотношение

$$
\begin{aligned}
& N_{l}\left(\bar{z}, u_{n-1}^{(1)}, \ldots, u_{n-1}^{(r)}\right)-\frac{l}{2^{r}}= \\
&=\frac{1}{2^{r}} \sum_{\left(c_{1}, \ldots, c_{r}\right) \neq \overline{0}}(-1)^{c_{1} z_{1}+\ldots+c_{r} z_{r}} \sum_{i=0}^{l-1}(-1)^{c_{1} u_{n-1}^{(1)}(i)+\ldots+c_{r} u_{n-1}^{(r)}(i)} .
\end{aligned}
$$

Оценим абсолютную величину левой части, разбивая правую часть на суммы по векторам $\bar{c}$, имеющим вес $k=1,2, \ldots, r$ :

$$
\left|N\left(\bar{z}, u_{n-1}^{(1)}, \ldots, u_{n-1}^{(r)}\right)-\frac{l}{2^{r}}\right| \leq \frac{1}{2^{r}} \sum_{k=1}^{r} \sum_{\left\{t_{1}, \ldots, t_{k}\right\} \subset \overline{1, r}}\left|\sum_{i=0}^{l-1}(-1)^{\sum_{s=1}^{k} u_{n-1}^{\left(t_{s}\right)}(i)}\right| .
$$


В силу (3), по аналогии с доказательством теоремы 7, справедливо равенство

$$
\left|\sum_{i=0}^{l-1}(-1)^{\sum_{s=1}^{k} u_{n-1}^{\left(t_{s}\right)}(i)}\right|=\left|\sum_{\left(j_{1}, \ldots, j_{k}\right) \in R^{k}} \mu_{j_{1}} \ldots \mu_{j_{k}} \sum_{i=0}^{l-1} \chi\left(v_{t_{1}, \ldots, t_{k}, j_{1}, \ldots, j_{k}}(i)\right)\right|,
$$

поэтому

$$
\left|\sum_{i=0}^{l-1}(-1)^{\sum_{s=1}^{k} u_{n-1}^{\left(t_{s}\right)}(i)}\right| \leq\left(\sum_{j=0}^{2^{n}-1}\left|\mu_{j}\right|\right)^{k} \max _{v \in L_{R}(F), v \neq(0)}\left|\sum_{i=0}^{l-1} \chi(v(i))\right| .
$$

Из равенства (13), теоремы 1, леммы 5 и формулы бинома Ньютона следует, что

$$
\begin{aligned}
& \mid N\left(\bar{z}, u_{n-1}^{(1)}, \ldots, u_{n-1}^{(r)}\right)-\frac{l}{2^{r}} \mid \leq \frac{1}{2^{r}} \sum_{k=1}^{r}\left(\begin{array}{l}
r \\
k
\end{array}\right)\left(\frac{2}{\pi} \ln \left(2^{n-1}\right)+1\right)^{k} \times \\
& \times\left(\frac{2}{\pi} \ln \left(\frac{4 t}{\pi}\right)+1\right) 2^{\frac{m}{2}+n+\nu-1}= \\
&=\left(\left(\frac{1}{\pi} \ln \left(2^{n-1}\right)+1\right)^{r}-\frac{1}{2^{r}}\right)\left(\frac{2}{\pi} \ln \left(\frac{4 t}{\pi}\right)+1\right) 2^{\frac{m}{2}+n+\nu-1}
\end{aligned}
$$

\section{Список литературы}

1. Кузьмин А.С., Нечаев А.А. Линейные рекуррентные последовательности над кольцами Галуа. - Алгебра и логика, 1995, т. 34, № 2, с. 169-189.

2. Кузьмин А.С., Куракин В.Л., Нечаев А.А. Псевдослучайные и полилинейные последовательности. - Труды по дискретной математике, 1997, т. 1, с. 139-202.

3. Кузьмин А.С., Нечаев А.А. Линейные рекуррентные последовательности над кольцами Галуа. - Успехи матем. наук, 1993, т. 48, № 1, с. 167-168.

4. Куракин В.Л. Первая координатная последовательность линейной рекурренты максимального периода над кольцом Галуа. - Дискретная математика, 1994, т. 6 , № 2, c. 88-100.

5. Кузьмин А.С. Распределение элементов на циклах линейных рекуррент над кольцами вычетов. - Успехи матем. наук, 1992, т. 47, № 6, с. 213-214.

6. Камловский О.В. Частотные характеристики линейных рекуррентных последовательностей над кольцами Галуа. - Матем. сборник, 2009, т. 200, № 4, c. 31-52.

7. Lathtonen L., Ling S., Sole P., Zinoviev D. $\mathbb{Z}_{8}$-Kerdock codes and pseudorandom binary sequences. - J. Complexity, 2004, v. 20, № 8, p. 318-330. 
8. Sole P., Zinoviev D. The most significant bit of maximum-length sequences over $\mathbb{Z}_{2^{l}}$ : autocorrelation and imbalance. - IEEE Trans. Inf. Theory, 2004, v. 50, № 8, p. $1844-1846$.

9. Sole P., Zinoviev D. Distribution of $r$-patterns in the most significant bit of a maximum length sequence over $\mathbb{Z}_{2^{l}}$. - Lect. Notes Comp. Sci., 2005, v. 3486, p. 275-281.

10. Fan $S$., Han $W$. Random properties of the highest level sequences of primitive sequences over $\mathbb{Z}_{2^{e}}$. - IEEE Trans. Inf. Theory, 2003, v. 49, № 6, p. 1553-1557.

11. Dai Z.-D., Ye D., Wang P., Fang G. Distribution of $r$-patterns in the highest level of $p$-adic sequences over Galois rings. - Proc. Golomb symp., USC, 2002.

12. Hu H., Feng $D$., $W u W$. Incomplete exponential sums over Galois rings with applications to some binary sequences derived from $\mathbb{Z}_{2^{t}}$. - IEEE Trans. Inf. Theory, 2006, v. 52, № 5, p. 2260-2265.

13. Kuzmin A.S., Nechaev A.A. Complete weight enumerators of generalized Kerdock code and related linear codes over Galois ring. - Discr. Appl. Math., 2001, v. 111, p. 117-137.

14. Morgan M. D. The distribution of second order linear recurrence sequences mod $2^{m}$. Acta Arith., 1998, v. 83, № 2, p. 181-195.

15. Qi W., Zhou J. Distribution of 0 and 1 in the highest level of primitive sequences over $\mathbb{Z}_{2^{e}}$. - Science in China (Series A), 1997, v. 40, № 6, p. 606-611.

16. Амбросимов А. С. О распределении мультиграмм в линейных рекуррентных последовательностях над кольцом вычетов. - Успехи матем. наук, 1993, т. 48, № 5, c. $157-158$.

17. Фихтенгольи Г. М. Курс дифференциального и интегрального исчисления. Т. 2. М.: Наука, 1966, с. 800.

18. Boztas S., Hammous R., Kumar P. V. 4-phase sequences with near-optimum correlation properties. - IEEE Trans. Inform. Theory, 1992, v. 38, № 3, p. 1101-1113.

19. Sarwate D. V. An upper bound on the aperiodic autocorrelation function for a maximal-length sequence. - IEEE Trans. Inform. Theory, 1984, v. 30, p. 685-687. 